\title{
Corrigendum and addendum to section 3 of "Level spacing functions and non linear differential equations"
}

\author{
G. Mahoux and M.L. Mehta \\ J. Phys. I. France 3 (1993) 697-715.
}

In the above reference we missed to note that the functions $\mathcal{A}, w, u, v$, and $i \xi$ in equations (3.11)-(3.21) are real for real $\tau$, so that the absolute value of $y_{a 2}(\tau)$ is unity for real $\tau$. In other words, if we set $y_{a 2}=\exp (2 i \rho), \rho(\tau)$ is real for real $\tau$, satisfies the (non linear) differential equation

$$
\left(\rho^{\prime \prime}+\frac{\rho^{\prime}+2}{\tau}\right) \tan \rho-\rho^{2}+4=0
$$

and near $\tau=0$ has the expansion

$$
\rho(\tau)=-2 \tau\left[1-\zeta \tau+O\left(\tau^{3}\right)\right]
$$

Thus equation (3.5) should be corrected as

$$
\begin{aligned}
y_{a 2} & =\exp \left[-4 i \tau+4 i \zeta \tau^{2}+O\left(\tau^{4}\right)\right] \\
& =1-4 i \tau+4(i \zeta-2) \tau^{2}+16\left(\zeta-\frac{2 i}{3}\right) \tau^{3}+O\left(\tau^{4}\right)
\end{aligned}
$$

Also it was shown that each of the four functions $\operatorname{Re} \mathcal{S}(\tau), \operatorname{Im} \mathcal{S}(\tau), \mathcal{A}(\tau), \mathcal{B}(\tau)$ can be expressed in terms of a fifth Painlevé transcendent (P5), and one of them, namely $\mathcal{B}(\tau)$, also in terms of a third Painlevé transcendent (P3). Actually, according to Gromak (see the reference below), each of the remaining three functions can also be expressed in terms of a P3. In other words, as the P5 with equation (3.5') is related to the P3 with equation (3.8), so the P5 with equations (3.2), (3.3) and (3.4) are related to the P3 with respectively

(1) $\alpha=-1, \beta=3, \gamma=1, \delta=-1$; and for $\tau \ll 1$,

$$
y(\tau)=-\frac{1}{\zeta}+\left(3-\frac{1}{\zeta^{2}}\right) \tau+\left(-\frac{1}{\zeta^{3}}+\frac{3}{\zeta}-2 \zeta\right) \tau^{2}+\ldots
$$

(2) $\alpha=-1, \beta=3, \gamma=1, \delta=-1$; and for $\tau \ll 1$,

$$
y(\tau)=\frac{\tau}{3}+\frac{\tau^{3}}{45}-\frac{\zeta \tau^{4}}{27}+\ldots
$$

and

(3) $\alpha=1, \beta=1, \gamma=1, \delta=-1$; and for $\tau \ll 1$, 


$$
y_{+}(\tau)=-\tau+\zeta \tau^{2}-\frac{\tau^{3}}{3}+\ldots
$$

or another possible choice

$$
y_{-}(\tau)=-\frac{1}{\tau}-\zeta-\left(\zeta^{2}-\frac{1}{3}\right) \tau+\ldots .
$$

Here are the necessary details. This addendum is supposed to be read with the original reference.

Consider the couple of equations

$$
f=\frac{\sqrt{\tau}}{R^{2}-\tau}\left(R^{\prime}-\frac{R}{\tau}\right), \quad R=-\frac{\sqrt{\tau}}{f^{2}-1}\left(f^{\prime}+\frac{3}{2} \frac{f}{\tau}\right) .
$$

Eliminating $f$ one gets equation (3.10), while eliminating $R$ one gets

$$
f^{\prime \prime}=\frac{f}{f^{2}-1} f^{\prime 2}-\frac{f^{\prime}}{\tau}-\frac{9}{4 \tau^{2}} \frac{f}{f^{2}-1}+f\left(f^{2}-1\right) .
$$

Set $f=\sqrt{u /(u-1)}$, or $u=f^{2} /\left(f^{2}-1\right)$, so that

$$
u^{\prime \prime}=\frac{3 u-1}{2 u(u-1)} u^{2}-\frac{u^{\prime}}{\tau}+\frac{9 u}{2 \tau^{2}}(u-1)^{2}-2 u \text {. }
$$

This is again almost a P5 with $\delta=0$, and becomes a standard P5 on taking $\tau^{2}$ as the new independent variable.

Consider now the couple of equations

$$
\frac{u+1}{u-1}=\omega^{2}-\omega^{\prime}-\frac{2 \omega}{\tau}, \quad \omega=\frac{2 \tau u}{\tau u^{\prime}-3 u(u-1)} .
$$

Eliminating $\omega$, one gets (3.36), while eliminating $u$ one gets a P3 for $\omega$ with $\alpha=-1, \beta=$ $3, \gamma=1, \delta=-1$.

In equation $(3.21)$ if one writes $\xi=\left(y^{2}-1\right) /(2 i y)$, then one gets

$$
y^{\prime \prime}=\frac{y^{\prime 2}}{y}-\frac{y^{\prime}}{\tau}+\frac{1}{\tau}\left(y^{2}+1\right)+y^{3}-\frac{1}{y},
$$

a P3 with $\alpha=1, \beta=1, \gamma=1, \delta=-1$. Expressing y in terms of $\xi$,

$$
y_{ \pm}=i \xi \pm \sqrt{1+(1 \xi)^{2}}
$$

one gets equation (3.32) or (3.33).

Some one-parameter families of solutions of these equations are known, but none of them satisfies our conditions at $\tau=0$.

\section{Reference}

Gromak V.I., Reducibility of Painlevé equations, Diff. Urav. 20 (1984)1674-1683. 